\title{
Cisto coloide no terceiro ventrículo e morte súbita em jovem
}

Primeira submissão em 20/06/10

Colloid cyst in the third ventricle and sudden death in young man

Angela Augusta Ferreira de Alencar'; Aristoteles Comte de Alencar Filho'; Luiz Carlos de Lima Ferreira ${ }^{3}$; Aristoteles Comte de Alencar Neto ${ }^{4}$

\section{unitermos}

Cistos coloides

Morte súbita

Hipertensão intracraniana

\section{resumo}

Morte súbita de jovem, sexo masculino, 23 anos, assintomático, suscitou verificação de óbito. Antes queixou-se de cefaleia excruciante, em sala de aula, caindo sobre o computador. Encéfalo apresentou edema e congestão vascular. Sem herniações. Cortes coronais evidenciaram dilatação dos ventrículos laterais e nódulo aderido ao teto do terceiro ventrículo. À microscopia o diagnóstico foi cisto coloide do terceiro ventrículo. A ameaça de morte súbita em portadores de cisto coloide é sério problema diagnóstico na emergência médica. A relevância deste caso está em lembrar aos médicos que esta entidade deve permanecer como diagnóstico diferencial nas cefaleias em crianças, adolescentes e adultos jovens.

\section{abstract}

Sudden death of 23 year-old asymptomatic male patient led to further investigation into its cause. Previously, he had complained of excruciating headache in the classroom, falling on the computer. His brain showed edema and vascular congestion without herniation. Coronal sections showed dilatation of the lateral ventricles and nodule attached to the roof of the third ventricle. The microscopic diagnosis was colloid cyst of third ventricle. The threat of sudden death among colloid cyst patients is a serious diagnostic problem in medical emergencies. The relevance of this case is to remind doctors that this entity should remain as differential diagnosis in headaches among children, adolescents and young adults. key words

Colloid cysts

Death sudden

Intracranial hypertension

1. Mestra em Educação; especialista em Patologia; médica legista do Instituto Médico-Legal (IML) de Manaus.

2. Doutorando em Medicina (Cardiologia) do Programa de Pós-Graduação em Medicina da Universidade Estadual Paulista (UNESP); especialista em Cardiologia; médico da Universidade Federal do Amazonas (UFAM); médico da Fundação Centro de Oncologia do Amazonas (FCECON).

3. Doutor em Anatomia Patológica; professor titular do Departamento de Patologia da UFAM.

4. Acadêmico do Curso de Medicina da UFAM. 


\section{Introdução}

A morte súbita é um acontecimento inesperado, de grande impacto social, principalmente quando ocorre em pessoas jovens, sem ter sido precipitada por causas violentas ou doença preexistente. É uma das situações mais delicadas com a qual pode se deparar o perito legista.

Durante uma necropsia, excepcionalmente pode ocorrer uma situação em que o indivíduo, vítima de morte súbita, não apresente registro de antecedentes patológicos, nem lesões orgânicas evidentes, além de não apresentar manifestações de agressão violenta. Esse fato registra informalmente o que se chama de "necropsia branca"(5). No entanto, se são diagnosticadas lesões orgânicas, mas essas alterações morfopatológicas não se mostram totalmente suficientes para explicar a morte, então com certeza estamos diante da situação mais complexa e difícil da perícia médico-legal, ainda mais quando não existe qualquer manifestação exógena que se possa atribuir como causa do óbito(5).

Os cistos coloides do terceiro ventrículo são tumores raros do sistema nervoso central (SNC) e reconhecidos como causa de morte inesperada em jovens, assim como em adultos saudáveis e crianças, e que apresentam dificuldades para o diagnóstico clínico(4). Os sintomas clínicos são inespecíficos, geralmente cefaleia intermitente e de duração variável. A patogênese da morte súbita, que pode ocorrer em portadores dessa lesão benigna disembriogênica, ainda não está totalmente esclarecida. Nem o tamanho do cisto, nem o grau de dilatação ventricular, ou a duração dos sintomas podem fornecer indicadores prognósticos dessa complicação potencialmente fatal(9).

O presente estudo relata um caso de morte súbita em jovem assintomático, no qual, por meio de necropsia, descobriu-se um cisto no terceiro ventrículo cerebral, e realiza revisão de casos existentes na literatura. Dessa forma, espera-se contribuir para o conhecimento dos aspectos clinicopatológicos dessa lesão.

\section{Relato de caso}

A morte súbita e inesperada de um jovem de 23 anos em sala de aula, diante do monitor do computador onde aprendia suas lições de informática, suscitou a solicitação de uma verificação de óbito ao Instituto Médico-Legal de Manaus, pela autoridade policial de plantão em uma delegacia. Colhido o relato supracitado com os familiares, foi informado ainda que o periciando queixou-se de cefaleia intensa imediatamente antes da morte e que não tinha nenhuma doença prévia, trabalhando como auxiliar de expediente em um turno e cursando informática em outro. Realizada ectoscopia do cadáver, não foram encontrados quaisquer sinais de violência ou de doença preexistente. Na necropsia, nenhuma alteração macroscópica significativa foi encontrada internamente após abertura das cavidades craniana, torácica e abdominal, resultando na chamada "necropsia branca". Diante do histórico de cefaleia excruciante antes do óbito e da presença de discreto edema e hiperemia do parênquima encefálico, optou-se pela retirada e posterior exame do encéfalo. A declaração de óbito foi preenchida como morte de causa indeterminada.

Posteriormente, ao exame macroscópico do encéfalo fixado em formol a $20 \%$, puderam-se observar edema e congestão vascular discretos do parênquima. Ausência de hérnias tentoriais ou das tonsilas cerebelares. Aos cortes coronais sucessivos observaram-se dilatação dos ventrículos laterais e presença de nódulo tumoral medindo $2 \times 1,5 \mathrm{~cm}$, de consistência branda, frouxamente aderido ao plexo coroide, no teto do terceiro ventrículo, ocluindo o forame de Monro (Figura 1). O quarto ventrículo apresentava-se colabado e sem dilatação.

A microscopia do nódulo evidenciou paredes císticas constituídas por tecido fibrocolagênico revestidas por epitélio pseudoestratificado ora cúbico, ora colunar ciliado, aderido a estruturas vasculares do plexo coroide, contendo material amorfo eosinofílico denso periodic acid-Schiff (PAS) positivo, de permeio a estruturas radiadas semelhantes a hifas e detritos celulares, concluindo-se pelo diagnóstico de cisto coloide do teto do terceiro ventrículo (Figuras 2 e 3 ).

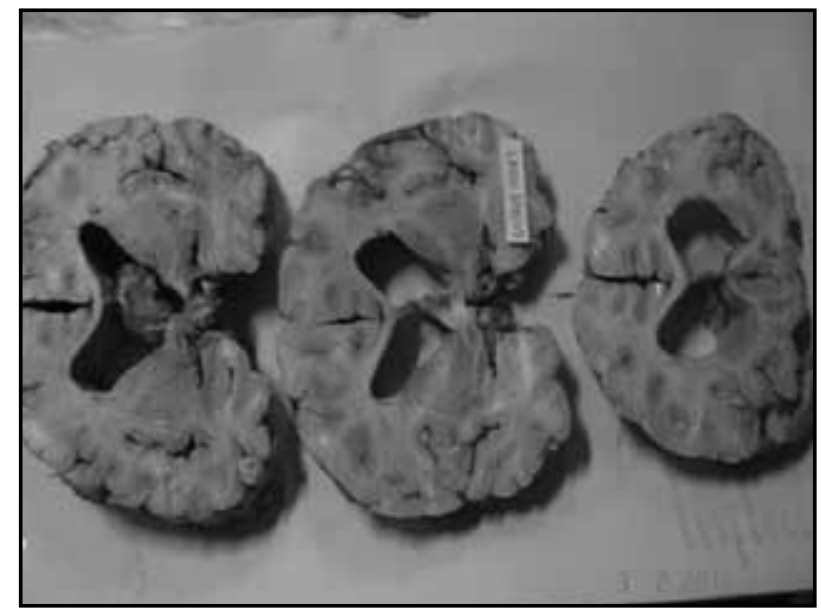

Figura 1 - Cortes coronais que mostram dilatação dos ventrículos laterais e presença de nódulo tumoral no teto do terceiro ventrículo ocluindo o forame de Monro 


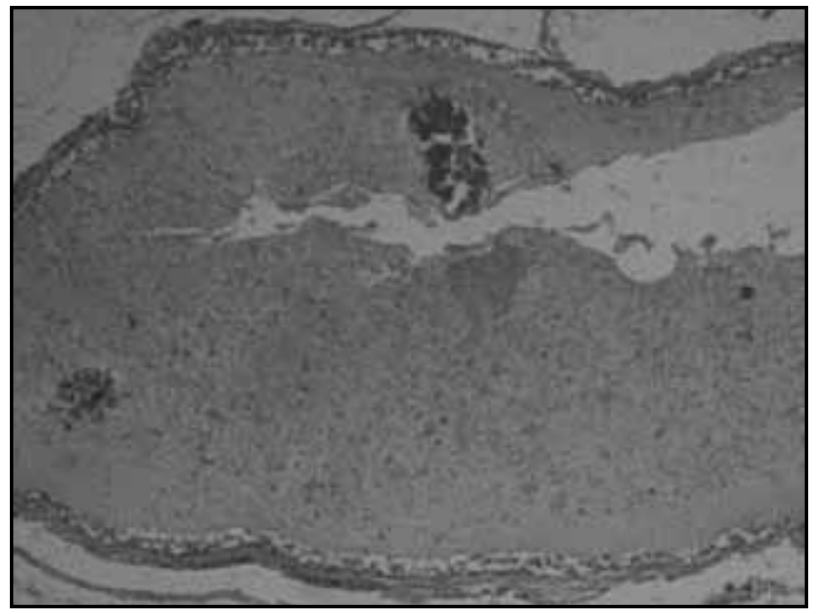

Figura 2 - Microscopia do nódulo que mostra paredes císticas constituídas por tecido fibrocolagênico revestidas por epitélio pseudoestratificado ora cúbico, ora colunar ciliado

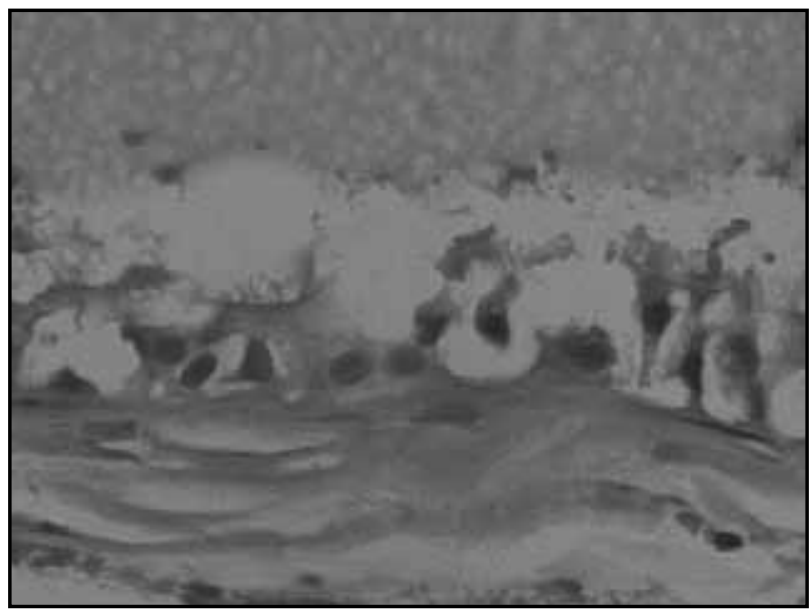

Figura 3 - Microscopia do nódulo que mostra revestimento por epitélio colunar ciliado

\section{Discussão}

Das várias lesões benignas do SNC, somente algumas são consideradas "verdadeiras", como, por exemplo, os cistos revestidos por epitélio. A principal dessas lesões é o cisto coloide anterossuperior do terceiro ventrículo. Investigações ultraestruturais e imuno-histoquímicas embasam sua origem em tecidos endodérmicos ectópicos ${ }^{(13)}$. São lesões raras, respondendo por $0,2 \%$ a $2 \%$ de todas as neoplasias intracraniais, tendo sido descrito pela primeira vez por Wallmann, em $1858^{(2)}$. São cistos benignos uniloculares de origem neuroepitelial que se apresentam aderidos na porção anterior do terceiro ventrículo ${ }^{(11)}$. É uma lesão benigna intracraniana contendo líquido mucoide que surge a partir de restos embrionários no teto do terceiro ventrículo. No presente caso a lesão encontrava-se no teto do terceiro ventrículo, ocluindo o forame de Monro e causando dilatação dos ventrículos laterais e colabamento do quarto ventrículo.
Existem evidências imuno-histoquímicas e ultraestruturais que favorecem a origem da lesão em restos endodérmicos, pois seu componente epitelial corresponde a células semelhantes às da mucosa respiratória, com uma camada de glicocálix apical particularmente característica de epitélios derivados do endoderma e que expressa citoqueratinas, antígeno da membrana epitelial e antígeno carcinoembrionário ${ }^{(3,13)}$.

A idade usual de diagnóstico ocorre da segunda à quinta década de vida, com predileção por homens. De forma coerente, no caso em tela, o achado foi em jovem do sexo masculino, aos 23 anos.

De crescimento lento, a lesão bloqueia eventualmente o forame de Monro e causa hidrocefalia obstrutiva. O cisto coloide pode ser assintomático, mas sintomas podem ser decorrentes do aumento da pressão intracraniana e secundários a hidrocefalia obstrutiva ${ }^{(8)}$.

Deterioração clínica aguda e morte súbita são complicações bem conhecidas de processos patológicos intracranianos. Elas são, na maioria das vezes, causadas por sangramento intracraniano ou intratumoral, hidrocefalia aguda e/ou herniações cerebrais( ${ }^{(6)}$. No presente, apesar da dilatação dos ventrículos laterais, não houve herniamentos ou sangramentos cerebrais.

A incidência de morte súbita causada por tumor cerebral varia de $0,16 \%$ a $3,2 \%$ e a maioria dos casos é devida a cisto coloide do terceiro ventrículo(6). O mecanismo de deterioração aguda e morte súbita é um processo dinâmico e multifatorial que inclui o grau e a duração de hipertensão intracraniana preexistente, a rapidez do aumento no volume intracraniano, a diminuição da complacência, a altura e duração das ondas de pressão e o aumento da pressão do seio sagital, o que provoca edema cerebral agudo seguido por uma série de eventos geralmente irreversíveis. Outros fenômenos, como resposta sistêmica, reações celulares e moleculares do cérebro, devem ser também considerados nas situações de estresse cerebral(6). O caso reportado apresentou-se com morte súbita do indivíduo precedida por cefaleia intensa, seguida de perda da consciência e resultando em queda sobre o computador em que trabalhava.

A morte súbita geralmente está associada às doenças cardiovasculares (DCVs), variando segundo o sexo e a idade e dependendo da etiologia da enfermidade. Entretanto, mesmo nos casos em que é realizada a necropsia, muitas vezes não é possível diagnosticar a causa exata, tanto pela falta de lesões histológicas, como no caso de infarto agudo do miocárdio (IAM) e arritmias cardíacas, e também pela possibilidade de outras patologias, como distúrbios 
hidroeletrolíticos, doenças metabólicas e/ou que necessitem de estudos mais complexos para serem diagnosticadas ${ }^{(12)}$.

A ameaça de morte súbita coloca os pacientes portadores de cisto coloide em sério problema diagnóstico na emergência médica. Eles podem apresentar discretos sinais e sintomas intermitentes e inespecíficos associados ao tumor $^{(10)}$. No presente caso a cefaleia excruciante e a morte súbita foram os únicos eventos evidenciados.

Existem controvérsias sobre a ocorrência familiar do cisto coloidal, porém o aumento do número de relatos de incidência familial de cisto coloide sugere a possibilidade de um perfil genético de herança dominante. Mas até esta data um padrão familial de hereditariedade não está reconhecido. Se existe uma ligação genética, o rastreamento de parentes assintomáticos pode estar indicado ${ }^{(1,8)}$.

Apesar de a deterioração e morte súbitas serem complicações conhecidas em pacientes portadores de tumores benignos do terceiro ventrículo, a exata incidência dessa catastrófica ocorrência não é bem conhecida ${ }^{(14)}$. A maioria dos cistos relatados na literatura é sintomática. O consenso atual é de que os cistos coloides sintomáticos devam ser tratados. Várias opções terapêuticas foram propostas para o seu tratamento com controle sequencial de imagens. A remoção endoscópica é considerada a terapia de escolha, devido aos resultados satisfatórios, à mínima taxa de complicações, ao tempo de hospitalização reduzido, ao retorno precoce ao trabalho e aos custos reduzidos $(7,11)$.

A relevância deste relato de caso está em lembrar aos médicos patologistas, legistas e de outras especialidades (clínicos, neurologistas, neurocirurgiões e urgentistas) que esta entidade, apesar de rara, deve permanecer como diagnóstico diferencial nas cefaleias em crianças, adolescentes e adultos jovens, e, em última hipótese, em achados de hidrocefalia durante necropsia.

\section{Referências}

1. AgGARWAL, A.; CORBETT, A. J. G. Familial colloid cyst of the third ventricle. J Clin Neurosci, v. 6, n. 6 , p. 520-2, 1999.

2. BÜTTNER, A. et al. Colloid cysts of the third ventricle with fatal outcome: a report of two cases and review of the literature. International Journal of Legal Medicine, v. 110 , n. 5, p. 260-6, 1997.

3. COCA, S. et al. Immunohistochemical study of intracranial cysts. Histol Histopathol, v. 8, n. 4, p. 651-4, 1993.

4. FILKINS, J. et al. Unexpected deaths due to colloid cysts of the third ventricle. J Forensic Sci, v. 41, n. 3, p. 512-3, 1996.

5. FRANÇA, G. V. Medicina legal. Rio de Janeiro: Guanabara Koogan, 2008.

6. HAMLAT, A.; PASQUALINI, E.; ASKAR, B. Hypothesis about the physiopathology of acute deterioration and sudden death caused by colloid cysts of the third ventricle. Medical Hypotheses, v. 63, n. 6, p. 1014-7, 2004.

7. JEFFREE, R. L., BESSER, M. Colloid cyst of the third ventricle: a clinical review of 39 cases. J Clin Neurosci, v. 8, n. 4, p. 328-31, 2001.
8. JOSHI, S. et al. A case of familial third ventricular colloid cyst. Emerg Med J, v. 22, n. 12, p. 909-10, 2005.

9. KUCHELMEISTER, K. Colloid cysts of the $3^{\text {rd }}$ ventricle: an underestimated danger? Neurochirurgia (Stuttg), v. 35, n. 1, p. 5-8, 1992.

10. MCDONALD, J. A. Colloid cyst of the third ventricle and sudden death. Annals of Emergency Medicine, v. 11, n. 7, p. 365-7, 1982.

11. PINTO, F. C. G. et al. Treatment of colloid cysts of the third ventricle through neuroendoscopic $\mathrm{Nd}$ : YAG laser stereotaxis. Arquivos de Neuro-Psiquiatria, v. 67, p. 1082-7, 2009.

12. REIS, L. M. D.; CORDEIRO, J. A., CURY, P. M. Análise da prevalência de morte súbita e os fatores de riscos associados: estudo em 2.056 pacientes submetidos a necropsia. Jornal Brasileiro de Patologia e Medicina Laboratorial, v. 42, p. 299-303, 2006.

13. ROSAI, J. Rosai and Ackermans surgical pathology. New York: Elsevier, 2004.

14. RYDER, J.; KLEINSCHMIDT-DEMASTERS, B.; LKELLER, T. Sudden deterioration and death in patients with benign tumors of the third ventricle area. J Neurosurg, v. 64, n. 2, p. 216-23, 1986. 